\title{
Age-related decline in mitochondrial DNA copy number in isolated human pancreatic islets
}

\author{
L. M. Cree • S. K. Patel • A. Pyle • S. Lynn • \\ D. M. Turnbull • P. F. Chinnery $\bullet$ M. Walker
}

Received: 6 February 2008 /Accepted: 30 April 2008 /Published online: 5 June 2008

(C) Springer-Verlag 2008

\begin{abstract}
Aim/hypothesis Pancreatic beta cell function has been shown to decline with age in man. Depletion of mitochondrial DNA (mtDNA) copy number is associated with impaired insulin secretion in pancreatic beta cell lines, and decreased mtDNA copy number has been observed with age in skeletal muscle in man. We investigated whether mtDNA copy number decreases with age in human pancreatic beta cells, which might in turn contribute to the age-related decline in insulin secretory capacity.

Methods We quantified mtDNA copy number in isolated human islet preparations from 15 pancreas donors aged between 17 and 75 years. Islets $(n=20)$ were individually
\end{abstract}

L.M. Cree and S.K. Patel contributed equally to this study.

L. M. Cree $\cdot$ A. Pyle $\cdot$ D. M. Turnbull $\cdot$ P. F. Chinnery

Mitochondrial Research Group, Faculty of Medical Sciences,

Newcastle University,

Newcastle upon Tyne, UK

S. K. Patel

Department of Medicine, Cardiovascular Research Group, University of Melbourne,

Melbourne, Australia

\section{S. Lynn}

Institute of Human Genetics, Faculty of Medical Sciences,

Newcastle University,

Newcastle upon Tyne, UK

\section{Walker $(\square)$}

Diabetes Research Group, School of Clinical Medical Sciences, Faculty of Medical Sciences, Newcastle University,

Medical School,

Framlington Place,

Newcastle upon Tyne NE2 4HH, UK

e-mail: mark.walker@ncl.ac.uk hand-picked and pooled from each donor isolate for the quantification of mtDNA copy number and deleted mtDNA $(\%)$, which were determined using real-time PCR methods. Results There was a significant negative correlation between mtDNA copy number and islet donor age $(r=-0.53$, $p=0.044)$. mtDNA copy number was significantly decreased in islet preparations from donors aged $\geq 50$ years $(n=8)$ compared with those aged $<50$ years $(n=7)$ (median [interquartile range]: 418 [236-503] vs 596 [554-729] mtDNA copy number/diploid genome; $p=0.032$ ). None of the islet preparations harboured high levels of deleted mtDNA affecting the major arc.

Conclusion/interpretation Given the correlation between mtDNA content and respiratory chain activity, the agerelated decrease in mtDNA copy number that we observed in human pancreatic islet preparations may contribute to the age-dependent decline in pancreatic beta cell insulin secretory capacity.

Keywords Ageing · Human islets · Mitochondrial DNA copy number $\cdot$ Mitochondrial DNA deletion $\cdot$ Mitochondrial DNA depletion $\cdot$ Pancreatic beta cell function

\author{
Abbreviations \\ mtDNA mitochondrial DNA \\ nt nucleotide
}

\section{Introduction}

A characteristic feature of type 2 diabetes is an increased prevalence with age, and the key driver is an age-related decline in pancreatic beta cell function. After adjusting for changes in whole-body insulin sensitivity, insulin secretion 
decreases with age in non-diabetic individuals [1] and contributes to the age-related deterioration in glucose tolerance in the general population [1]. Furthermore, both glucose-stimulated insulin secretion and ATP content have been shown to decline with age in human pancreatic islets isolates [2]. What are the mechanisms that account for the age-related decline in pancreatic beta cell function?

Insulin secretion is highly energy dependent, requiring optimal ATP synthesis by an intact mitochondrial respiratory chain. Thirteen essential respiratory chain proteins are synthesised from small circles of double-stranded DNA present within each mitochondrion: the mitochondrial genome, or mtDNA. Both qualitative (mutation) and quantitative (depletion) defects of mtDNA have been implicated in the pathogenesis of diabetes. Specific point mutations and deletions of mtDNA are known to cause diabetes in man [3], while extreme mtDNA depletion decreased insulin secretion in the MIN6 cell line [4]. An age-related decline in mtDNA copy number has been reported in skeletal muscle from healthy individuals $[5,6]$, and was associated with a decrease in mitochondrial respiratory chain sub-unit expression and ATP production [5].

This raises the question of whether mitochondrial DNA copy number decreases with age in human pancreatic beta cells, and whether this in turn contributes to the age-related decline in insulin secretory capacity in man. As a step towards addressing this question, the aim of this study was to investigate whether there is evidence of an age-related decrease in mtDNA copy number in human pancreatic islets from non-diabetic donors aged between 17 and 75 years.

\section{Methods}

Human islet preparations Human islet preparations were obtained from the Leicester University Transplantation Facility, UK, between September 1997 and March 2000. The characteristics of the donor individuals are shown in Table 1. An aliquot of the islet preparation was removed and stained with dithizone $(25 \mu \mathrm{g} / \mathrm{ml})$ to assess purity and assist islet identification. Using the remainder of the islet preparation, individual islets were then hand-picked using a micromanipulator and stored at $-40^{\circ} \mathrm{C}$. The study was approved by the Newcastle and North Tyneside Local Research Ethics Committee.

$m t D N A$ copy number assay Stored individual islets $(n=20)$ from the same donor were lysed using a $0.5 \mathrm{~mol} / \mathrm{l}$ Tris $\mathrm{HCl}$ and $100 \mathrm{mmol} / \mathrm{l}$ EDTA buffer containing Proteinase $\mathrm{K}$ (Invitrogen, Paisley, UK) and then pooled. Mitochondrial DNA copy number was determined by real-time PCR using iQ SYBR Green on the BioRad iCycler iQ5 (BioRad, Hercules, CA, USA) to a target template spanning from
Table 1 Human islet donor preparation details

\begin{tabular}{llccc}
\hline $\begin{array}{l}\text { Donor } \\
\text { ID }\end{array}$ & $\begin{array}{l}\text { Recorded cause of donor } \\
\text { death }\end{array}$ & $\begin{array}{l}\text { Purity of islets } \\
(\%)\end{array}$ & Age & Sex \\
\hline F & RTC-head injury & 30 & 17 & M \\
G & MI & 30 & 56 & M \\
H & SAH & 90 & 49 & F \\
J & RTC & 70 & 25 & F \\
K & IC bleed & 70 & 62 & F \\
L & Cerebral oedema & 65 & 55 & F \\
M & SAH & 90 & 56 & M \\
N & IC bleed & 85 & 49 & - \\
P & SAH & 85 & 34 & F \\
R & MI & 85 & 68 & F \\
T & Head injury & 90 & 34 & M \\
U & SAH & 75 & 53 & F \\
V & RTC & 80 & 54 & M \\
W & SAH & 75 & 43 & F \\
Y & SAH & 70 & 75 & M \\
\hline
\end{tabular}

IC, intracranial bleed; F, female; M, male; MI, myocardial infarction; RTC, road traffic collision; SAH, subarachnoid haemorrhage

nucleotide (nt) 3459 to nt 3569 of the mtDNA ND1 gene (forward: ACGCCATAAAACTCTTCACCAAAG; reverse: GGGTTCATAGTAGAAGAGCGATGG) [7]. This was normalised using a nuclear-encoded template for the GAPDH gene spanning from nt 804 to nt 903 (NCBI, NM_002046.2). Relative copy number was calculated from the threshold cycle value $\left(\Delta C_{\mathrm{t}}\right.$ value), where the mean amount of mtDNA/cell $=2\left(2^{-\Delta \mathrm{C}_{\mathrm{t}}}\right)$, to account for the two copies of GAPDH in each cell nucleus. Each reaction was optimised and confirmed linear over an appropriate concentration range using genomic DNA standards. Samples were analysed in triplicate for both assays, enabling calculation of the average mtDNA:nuclear DNA (nDNA) ratio.

mtDNA deletion assay The proportion of mutated mtDNA was determined by real-time PCR [7] using iQ SYBR Green on the BioRad iCycler iQ5 (BioRad) to a target template spanning from nt 11144 to $n t 11250$ of the ND4 gene in the major arc of mtDNA that is the most common region to be deleted in pathogenic mtDNA deletions (forward: ACCTTGGCTATCATCACCCGAT; reverse: AGTGCGAT GAGTAGGGGAAGG). Each assay was performed in quadruplicate. The proportion of mutated mtDNA was then calculated from the ND4 (wild-type molecules) and ND1 (total mtDNA) data as described. This assay has a $20 \%$ detection threshold for deleted mtDNA [7].

Statistical analysis Data were analysed using Minitab version 14 (Minitab, State College, PA, USA). Spearman rank correlation was used to examine the correlation between mtDNA copy number and islet donor age. The Mann-Whitney $U$ test was used to compare mtDNA copy 
number between age groups. A $p$ value of $<0.05$ was considered statistically significant.

\section{Results}

Table 1 shows the characteristics of the islet donors. There was a wide age range with the youngest being 17 and oldest 75 years of age.

Figure 1 shows that there was a significant inverse correlation between mtDNA copy number and islet donor age in the pooled islets $(r=-0.53, p=0.044)$. mtDNA copy number in pooled islets was then compared between donors aged $<50$ years $(n=7)$ and those aged $\geq 50$ years $(n=8)$. mtDNA copy number was significantly less in those aged $\geq$ 50 years (median [interquartile range]: 418 [236-503] vs 596 [554-729] mtDNA copy number/diploid genome; $p=0.032$ ).

None of the islet preparations harboured high levels of deleted mtDNA affecting the major arc.

\section{Discussion}

To our knowledge, this is the first description of an agerelated decline in mtDNA copy number in isolated human islets. Some workers [5, 6], but not all [8,9], have observed a similar decrease in mtDNA copy number with age in skeletal muscle in man. Short and colleagues showed that the age-related decline in mtDNA copy number was associated with a decrease in mitochondrial ATP production and in cytochrome $c$ oxidase sub-unit expression [5]. This raised the possibility that the decrease in mtDNA copy number contributed directly to the decreased mitochondrial function. Goodpaster and colleagues also observed a decrease in mtDNA copy number in skeletal muscle from healthy, elderly individuals [6], but showed that exercise improved both mtDNA copy number and mitochondrial respiratory chain function in these individuals.

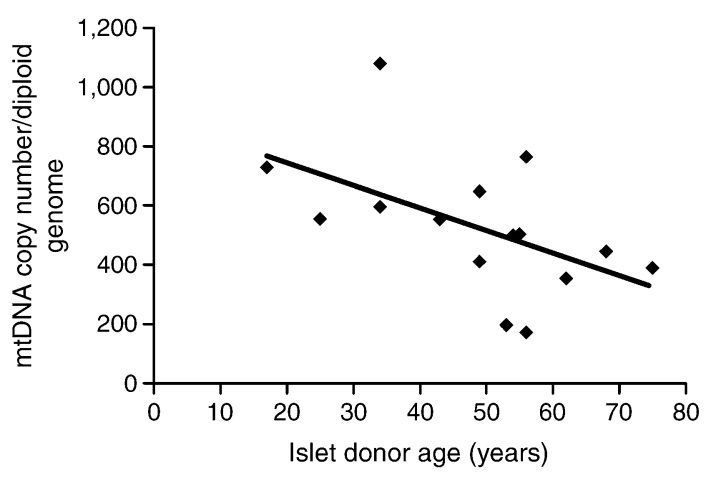

Fig. 1 mtDNA copy number with age using human pancreatic islets from 15 donor patients (results show mean mtDNA copy number from 20 pooled islets). $r=-0.53, p=0.044$
What might be the mechanism by which mtDNA copy number declines with age? The observed decrease in mtDNA copy number in skeletal muscle might simply result from an age-dependent decrease in physical activity, but such a mechanism is unlikely to explain the age-related decline in mtDNA copy number in human islets. The mitochondrial theory of ageing proposes that reactive oxygen species generated by the mitochondria lead to accumulative oxidative damage to mtDNA and a decline in mitochondrial function with age. Short and colleagues found that the age-related decline in mtDNA copy number in skeletal muscle was accompanied by an increase in the levels of 8-oxo-deoxyguanosine, a marker of global oxidative DNA damage [5]. A common site for age-related damage to the mtDNA genome is the major arc, which results in the generation of deleted mtDNA molecules. Work by our group has shown that the amount of deleted mtDNA as a percentage of the total mtDNA molecules increases with age in human substantia nigra neurons [10]. The mean level of deleted mtDNA was $43 \%$ in the neurons from aged brains. We therefore examined whether the observed age-related decline in mtDNA copy number in human islets was associated with an increased accumulation of deleted mtDNA molecules. None of the human islet preparations had a percentage of deleted mtDNA above $20 \%$, which was the detection level of the assay under our study conditions. It may be that human islets, compared with neurons of the substantia nigra, are more resistant to damage, or that islets cells with high levels of deleted mtDNA are selectively removed, as is suspected for islet cells with the $3243 \mathrm{~A}>\mathrm{G}$ myopathy encephalopathy, lactic acidosis and stroke-like episodes (MELAS) mutation [11].

Does the age-related decline in mtDNA copy number affect beta cell function directly, or is it simply a biomarker of the ageing process? We know that extreme mtDNA depletion in cultured MIN6 cells decreases glucose-stimulated insulin secretion and that re-population of the cells with mtDNA reverses the defect [4]. As shown in Fig. 1, however, mean mtDNA copy number fell by about $50 \%$ between 17 and 75 years of age, raising the question of whether lesser degrees of mtDNA depletion affect beta cell function directly. We were unable to conduct concurrent functional studies, but it is intriguing to note that a similar study of human islet isolates reported an age-related decline in both glucose-stimulated insulin secretion and islet ATP content [2].

A potential criticism of our work is that we have studied human islets that have undergone and survived the standard isolation process. We do not know whether islets with lower levels of mtDNA are more susceptible to damage and hence are lost during the isolation process. However, if this was the case then it might be argued that the islet preparations from older donors may be more susceptible 
to this effect and that we may have underestimated the agerelated decline in mtDNA copy number.

In conclusion, we have demonstrated an age-related decline in mtDNA copy number in whole human islets. As these comprise a number of different cell types [12], the next step will be to determine whether an age-related decline in mtDNA copy number results directly in both decreased mitochondrial function and insulin secretion in individual pancreatic beta cells.

Acknowledgements This work was funded by Diabetes UK. P. F. Chinnery is a Wellcome Trust Senior Fellow in Clinical Science. P. F. Chinnery also receives funding from the Parkinson's Disease Society (UK), the United Mitochondrial Diseases Foundation, and the EU FP programme EUMITOCOMBAT and MITOCIRCLE.

Duality of interest The authors declare that there is no duality of interest associated with this manuscript.

\section{References}

1. Basu R, Breda E, Oberg AL et al (2003) Mechanisms of the ageassociated deterioration in glucose tolerance; contribution of alterations in insulin secretion, action, and clearance. Diabetes 52:1738-1748

2. Ihm S-H, Matsumoto I, Sawada T et al (2006) Effect of donor age on function of isolated human islets. Diabetes 55:1361-1368
3. Maasen JA, Hart LM, Essen E et al (2004) Mitochondrial diabetes. Diabetes 53(Suppl 1):103-109

4. Soejima A, Inoue K, Takai D et al (1996) Mitochondrial DNA is required for regulation of glucose-stimulated insulin secretion in a mouse pancreatic cell line, MIN6. J Biol Chem 271:26194-26199

5. Short KR, Bigelow ML, Kahl J et al (2005) Decline in skeletal muscle mitochondrial function with aging in humans. Proc Natl Acad Sci U S A 102:5618-5623

6. Menshikova EV, Ritov VB, Fairfull L, Ferrell RE, Kelley DE, Goodpaster BH (2006) Effects of exercise on mitochondrial content function in aging human skeletal muscle. J Gerontol 61:534-540

7. He L, Chinnery PF, Durham SE et al (2002) Detection and quantification of mitochondrial DNA deletions in individual cells by real-time PCR. Nucleic Acids Res 30:e68

8. Miller FJ, Rosenfeldt FL, Zhang C, Linnane AW, Nagely P (2003) Precise determination of mitochondrial DNA copy number in human skeletal and cardiac muscle by a PCR-based assay: lack of change of copy number with age. Nucleic Acids Res 31:e61

9. Frahm T, Mohamed SA, Bruse P, Gemund C, Oehmichen M, Meissner C (2005) Lack of age-related increase of mitochondrial DNA amount in brain, skeletal muscle and human heart. Mech Ageing Dev 126:1192-1200

10. Bender A, Krishnan KJ, Morris CM et al (2006) High levels of mitochondrial DNA deletions in substantia nigra neurons in aging and Parkinson disease. Nat Genet 38:515-517

11. Lynn S, Borthwick GM, Charnley RM, Walker M, Turnbull DM (2003) Heteroplasmic ratio of the A3243G mitochondrial DNA mutation in single pancreatic beta cells. Diabetologia 46:296-299

12. Muller D, Huang GC, Amiel S, Jones PM, Persuad SJ (2007) Gene expression heterogeneity in human islet endocrine cells in vitro: the insulin signalling cascade. Diabetologia 50:1239-1242 